\title{
The parallel projection operators of a nonlinear feedback system ${ }^{1}$
}

\author{
John C. Doyle ${ }^{2}$, Tryphon T. Georgiou ${ }^{3}$, Malcolm C. Smith
}

\begin{abstract}
This paper defines and studies a pair of nonlinear parallel projection operators associated with a nonlinear feedback system. These operators have been seen to play an important role in the robustness and design of linear systems especially in the theory of the gap metric, the use of weighted gaps in control system design and Glover-McFarlane loopshaping. We report the following results which are established in the full version of the paper [4]. The input-output $L_{2}$-stability of a feedback system is equivalent to a "coordinatization" of the input and output spaces, which is also equivalent to the existence of a pair of nonlinear parallel projection operators onto the graph of the plant and the inverse graph of the controller respectively. These projections have equal norms whenever one of the feedback elements is linear. A bound on this norm is given in the case of passive systems with unity negative feedback.
\end{abstract}

\section{Introduction}

This paper is based on [4] and studies a pair of nonlinear parallel projection operators associated with a nonlinear feedback system. The existence of these operators is guaranteed by the input-output $L_{2}$ stability of the feedback system (Propositions 3,4). The main result reported in the paper is that the projections have equal norms whenever one of the feedback elcments is linear (Proposition 6). This result was established for linear systems in [9] using coprime fraction techniques, and in [7] using operator theoretic techniques, and shown to imply a symmetry

\footnotetext{
${ }^{1}$ Supported in part by the NSF and the AFOSR, U.S.A., and the Nuffield Foundation, U.K.

${ }^{2}$ Department of Electrical Engineering, California Institute of Techuology, Pasadena, CA 91125 , U.S.A.

${ }^{3}$ Department of Electrical Engineering, University of Minnesota, Minneapolis, MN 55455, U.S.A.

${ }^{4}$ Department of Engineering, University of Cambridge, Cambridge, CB2 1PZ, U.K.
}

property regarding the tolerance of plant and controller uncertainty.

In the case of a stable linear feedback system in the form of Fig. 1 the relevant parallel projections are

$$
\begin{aligned}
& \Pi_{1}=\left(\begin{array}{l}
\mathbf{I} \\
\mathbf{P}
\end{array}\right)(\mathbf{I}-\mathbf{C P})^{-1}\left(\begin{array}{ll}
\mathbf{I} & -\mathrm{C}),
\end{array}\right. \\
& \Pi_{2}=\left(\begin{array}{l}
\mathbf{C} \\
\mathbf{I}
\end{array}\right)(\mathbf{I}-\mathbf{P C})^{-1}\left(\begin{array}{ll}
-\mathbf{P} & \mathbf{I}
\end{array}\right) .
\end{aligned}
$$

The quantity $\left\|\Pi_{1}\right\|^{-1}\left(=\left\|\Pi_{2}\right\|^{-1}\right)$ is the stability margin for uncertainty of $\mathbf{P}$ measured either by the gap metric or by perturbations of the normalized coprime factors of $P$ ([9], [12], [23]). In addition, the elements of $\Pi_{1}$ and $\Pi_{2}$ consist of important closed-loop transfer functions, such as the sensitivity, complementary sensitivity, control weighting etc. whose norms must be kept within reasonable bounds for good performance. The minimization (of a weighted version) of this measure has been exploited for controller design with various interpretations in [15], [13], [6], [1]. In [15] the weighting procedure is related to the open-loop loop shape, while in [6] and [1] the emphasis is on closed-loop trade-offs and uncertainty in the weighted gap.

It is evidently interesting to study possible generalizations of (1) and (2), and associated properties, to the nonlinear case. The approach taken here will make extensive use of the graphs of the plant and controller. In the context of linear control systems the "graph viewpoint" became especially prevalent after the introduction of the gap metric [27] and the subsequent progress on issues of robust stabilization against graph perturbations (e.g. [24], [22] [5], [23], [8], [12], and [9]), though the idea of studying systems via graph representations can be traced back as far as [21]. The graph viewpoint has been advanced in the context of nonlinear systems for obtaining abstract representations of such systems for stability analysis and robustness studies in [18] and [20]. An earlier relevant reference where a functional theoretic framework for studying 


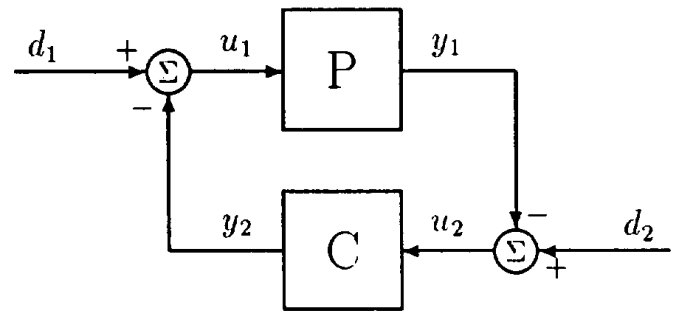

Figure 1: Standard feedback configuration.

stability of feedback systems was proposed is [26]. Recent works on operator theoretic and geometric views of stability, coprime factorizations and the gap metric include [2], $[7],[11],[16],[17],[19],[25],[28]$.

Before we formally define a nonlinear parallel projection we will show, using block diagrams, how these operators arise, and highlight some of their properties. Consider the feedback configuration of Fig. 1 where $\mathbf{P}$ and $\mathbf{C}$ are general nonlinear operators satisfying $\mathbf{P} 0=0$ and $\mathbf{C} 0=0$. Let the feedback configuration be stable, namely, let arbitrary $L_{2}$ signals $d_{1}$ and $d_{2}$ give rise to unique $L_{2}$ signals $u_{1}, u_{2}$, $y_{1}$ and $y_{2}$. Now define the operators

$$
\boldsymbol{\Pi}_{1}\left(\begin{array}{c}
d_{1} \\
d_{2}
\end{array}\right)=\left(\begin{array}{l}
u_{1} \\
y_{1}
\end{array}\right), \quad \Pi_{2}\left(\begin{array}{l}
d_{1} \\
d_{2}
\end{array}\right)=\left(\begin{array}{c}
y_{2} \\
u_{2}
\end{array}\right) .
$$

We first note from the feedback equations that

$$
\Pi_{1}+\Pi_{2}=\mathbf{I}
$$

It is also true that

$$
\Pi_{i}^{2}=\Pi_{i}
$$

for $i=1,2$. To see this observe, that if $u_{1}, u_{2}, y_{1}$ and $y_{2}$ are any $L_{2}$ signals satisfying $y_{1}=\mathbf{P} u_{1}$ and $y_{2}=\mathbf{C} u_{2}$, then the feedback equations are uniquely solved with external inputs $\left(\begin{array}{l}d_{1} \\ d_{2}\end{array}\right)=\left(\begin{array}{l}u_{1} \\ y_{1}\end{array}\right)$ and $\left(\begin{array}{l}d_{1} \\ d_{2}\end{array}\right)=\left(\begin{array}{l}y_{2} \\ u_{2}\end{array}\right)$ as in the feedback configurations of Fig. 2 and Fig. 3 respectively.

\section{Notation}

We consider a general nonlinear system to be a (possibly unbounded) operator $\mathbf{P}: \mathcal{D}_{\mathbf{P}} \subset \mathcal{U}-\mathcal{Y}$ where the domain $\mathcal{D}_{\mathbf{P}} \subset \mathcal{U}:=\mathcal{L}_{2}^{m}[0, \infty)$ and $\mathcal{Y}:=\mathcal{L}_{2}^{p}[0, \infty)$. Throughout, the underlying spaces will be assumed to be real. For any $u \in \mathcal{D}_{\mathbf{P}}$ we denote by $\mathbf{P} u$ the action of $\mathbf{P}$ on $u$. We assume

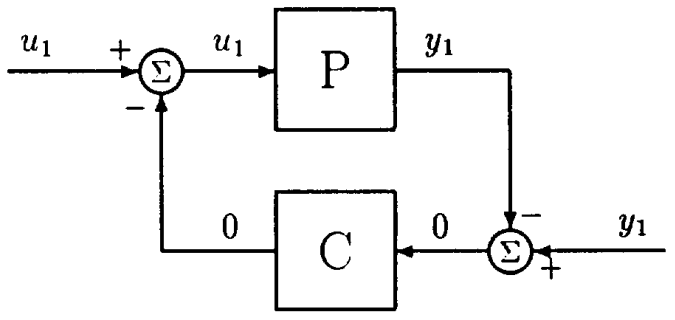

Figure 2: Standard feedback configuration-external inputs in plant graph.

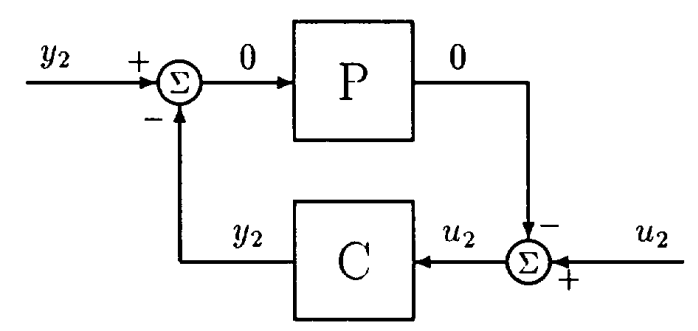

Figure 3: Standard feedback configuration-external inputs in controller graph.

throughout that $0 \in \mathcal{D}_{\mathbf{P}}$ and also that $\mathbf{P} 0=0$. The class of all such systems will be denoted by $\mathcal{P}_{\mathfrak{u}, \mathcal{y}}$.

The graph of a given system $\mathbf{P} \in \mathcal{P}_{\mathfrak{u}, \mathcal{Y}}$ is the nonlinear manifold

$$
\mathcal{G}_{\mathbf{P}}:=\left(\begin{array}{c}
\mathbf{I}_{m} \\
\mathbf{P}
\end{array}\right) \mathcal{D}_{\mathbf{P}} \subset \mathcal{U} \times \mathcal{Y}=: \mathcal{L}
$$

A system $\mathbf{P} \in \mathcal{P}_{u, y}$ is said to be stable if $\mathcal{D}_{\mathbf{P}}=U$, and finite-gain ( $f g$ ) stable if $\mathbf{P}$ is stable and

$$
\|\mathbf{P}\|:=\sup _{0 \neq x \in l} \frac{\|\mathbf{P} x\|}{\|x\|}
$$

is finite.

Consider the ferdback configuration of Fig. 1 where the plant $\mathrm{P} \in \mathcal{P}_{\mathfrak{u}, \boldsymbol{y}}$ and the controller $\mathrm{C} \in \mathcal{P}_{\boldsymbol{y}, \boldsymbol{u}}$. This configuration, hereby denoted by $[\mathbf{P}, \mathbf{C}]$, gives a pictorial representation to the following set of equations:

$$
\begin{aligned}
& u_{1}=d_{1}-\mathbf{C} u_{2} \\
& u_{2}=-\mathbf{P} u_{1}+d_{2} .
\end{aligned}
$$

Define the (nonlinear) mapping

$\mathbf{F}_{\mathbf{P}, \mathbf{C}}: \mathcal{D}_{\mathbf{P}} \times \mathcal{D}_{\mathbf{C}} \rightarrow \mathcal{L}:\left(\begin{array}{l}u_{1} \\ u_{2}\end{array}\right) \rightarrow\left(\begin{array}{l}d_{1} \\ d_{2}\end{array}\right)=\left(\begin{array}{cc}\mathbf{I}_{m} & \mathbf{C} \\ \mathbf{P} & \mathbf{I}_{p}\end{array}\right)\left(\begin{array}{l}u_{1} \\ u_{2}\end{array}\right)$. 
The interconnection $[\mathbf{P}, \mathbf{C}]$ is said to be stable if $\mathbf{F}_{\mathbf{P}, \mathbf{C}}$ has a stable inverse, i.e., $\mathbf{F}_{\mathbf{P}, \mathbf{C}}^{-1} \in \mathcal{P}_{\mathcal{L}, \mathcal{L}}$. Similarly, $[\mathbf{P}, \mathbf{C}]$ is said to be fg-stable if the corresponding propery is true for $\mathbf{F}_{\mathrm{P}, \mathrm{C}}^{-1}$.

\section{Nonlinear parallel projections}

In this section we will present the main results of the paper. We first introduce the notion of coordinatization of a space. This is a generalization of the idea of a direct sum decomposition to the case of nonlinear manifolds. Proposition 4 states that a feedback system is stable if and only if the graph of the plant and the inverse graph of the controller define a coordinatization of the product space of inputs and outputs. We present a general notion of (nonlinear) parallel projection (Definition 2) which formalizes the properties of the operators $\Pi_{1}$ and $\Pi_{2}$ given in section 1 equation (3). Proposition 3 states that, in general, the existence of a coordinatization is equivalent to the existence of certain canonical parallel projections. For a stable feedback system these projections are the operators $\Pi_{1}$ and $\Pi_{2}$ of equation (3). These projections have equal norms whenever one of the feedback elements is linear (Proposition 6). A bound on this norm is presented for passive systems with unity negative feclback in Proposition 7.

Let $\mathcal{M}, \mathcal{N}$ be two (nonlinear) submanifolds of a given linear space $\mathcal{L}$ which include the origin. Typically, $\mathcal{L}=$ $\mathcal{L}_{2}^{m+p}[0, \infty)=U \times \mathcal{Y}$. However, for much of the discussion in this section $\mathcal{L}$ can be taken to be any (real) Ililbert space.

Definition 1. The pair $(\mathcal{M}, \mathcal{N})$ is said to induce a coordinatization of $\mathcal{L}$ if

(a) $\mathcal{L}=\mathcal{M}+\mathcal{N}$.

(b) $x_{\mathcal{M}}+x_{\mathcal{N}}=x_{\mathcal{M}}^{\prime}+x_{\mathcal{N}}^{\prime}$, with $x_{\mathcal{M}}, x_{\mathcal{M}}^{\prime} \in \mathcal{M}$ and $x_{\mathcal{N}}, x_{\mathcal{N}}^{\prime} \in \mathcal{N}$, implies that $x_{\mathcal{M}}=x_{\mathcal{M}}^{\prime}$ and $x_{\mathcal{N}}=x_{\mathcal{N}}^{\prime}$.

The following proposition gives alternate expressions for the second of the above conditions.

Proposition 1. Given $\mathcal{M}, \mathcal{N} \subset \mathcal{L}$, with $\mathcal{M} \cap \mathcal{N} \supset\{0\}$, the following statements are equivalent to Definition 1(b):

$\left(b_{1}\right)$ For any $x_{1}, x_{2} \in \mathcal{M}$, with $x_{1} \neq x_{2},\left\{x_{1}+\mathcal{N}\right\} \cap\left\{x_{2}+\right.$ $\mathcal{N}\}=\emptyset$.

(b2) For any $x_{1}, x_{2} \in \mathcal{N}$, with $x_{1} \neq x_{2},\left\{x_{1}+\mathcal{M}\right\} \cap\left\{x_{2}+\right.$ $\mathcal{M}\}=\emptyset$.
Proof. See [4].

It is immediate to see that in case $\mathcal{M}, \mathcal{N}$ are linear manifolds, then condition (b) is equivalent to $\mathcal{M} \cap \mathcal{N}=\{0\}$.

Definition 2. A stable operator $\Pi: \mathcal{L} \rightarrow \mathcal{L}$ (with $\Pi 0=$ 0 ) is said to be a parallel projection if for any $x_{1}, x_{2} \in$ $\mathcal{L}, \Pi\left(\Pi x_{1}+\left(\mathbf{I}_{\mathcal{L}}-\Pi\right) x_{2}\right)=\Pi x_{1}$, where $\mathbf{I}_{\mathcal{L}}$ denotes the identity on $\mathcal{L}$.

The condition given above requires a "distributivity" of $\Pi$ with respect to the additive decomposition $\mathcal{L}=\Pi \mathcal{L}+$ $\left(\mathbf{I}_{\mathcal{L}}-\mathbf{I}\right) \mathcal{L}$.

Proposition 2. If $\Pi$ is a parallel projection then

(a) $\Pi \Pi=\Pi$.

(b) $\left.\Pi\right|_{\text {range }(\Pi)}=\mathbf{I}_{\text {range }(\Pi)}$, where range $\Pi(=\mathbf{\Pi} \mathcal{L})$ denotes the range of $\boldsymbol{\Pi}$.

(c) $\boldsymbol{\Pi}\left(\mathbf{I}_{\mathcal{L}}-\boldsymbol{\Pi}\right)=\mathbf{0}$.

(d) $\mathbf{I}_{\mathcal{L}}-\Pi$ is a parallel projection.

Proof. Conditions (a) and (c) follow from the definition of a parallel projection when we set $x_{2}=0$ and $x_{1}=0$ respectively. Clearly (b) is equivalent to (a). The following computation establishes (d).

$$
\begin{aligned}
\left(\mathbf{I}_{\mathcal{L}}-\Pi\right)\left(\Pi x_{1}+\left(\mathbf{I}_{\mathcal{L}}-\Pi\right) x_{2}\right) \\
\quad=\Pi x_{1}+\left(\mathbf{I}_{\mathcal{L}}-\Pi\right) x_{2}-\Pi\left(\Pi x_{1}+\left(\mathbf{I}_{\mathcal{L}}-\Pi\right) x_{2}\right) \\
=\Pi x_{1}+\left(\mathbf{I}_{\mathcal{L}}-\Pi\right) x_{2}-\Pi x_{1} \\
=\left(\mathbf{I}_{\mathcal{L}}-\Pi\right) x_{2} . \square
\end{aligned}
$$

Proposition 3. If $I I$ is a parallel projection and $\mathcal{M}:=$ $\Pi \mathcal{L}, \mathcal{N}:=\left(\mathbf{I}_{\mathcal{L}}-\Pi\right) \mathcal{L}$, then $\mathcal{M}, \mathcal{N}$ induce a coordinatization of $\mathcal{L}$. Conversely, if the manifolds $\mathcal{M}, \mathcal{N} \subset \mathcal{L}$ induce a coordinatization of $\mathcal{L}$, then the canonical mappings $\Pi_{\mathcal{M} \| \mathcal{N}}: x \rightarrow x_{\mathcal{M}}$ and $\Pi_{\mathcal{N} \| \mathcal{M}}: x \rightarrow x_{\mathcal{N}}$, where $x=x_{\mathcal{M}}+x_{\mathcal{N}}$, with $x_{\mathcal{M}} \in \mathcal{M}$ and $x_{\mathcal{N}} \in \mathcal{N}$, define parallel projections.

Proof. $(\Rightarrow)$. Any $x \in \mathcal{L}$ can be written as $x=x_{\mathcal{M}}+x_{\mathcal{N}}$ with $x_{\mathcal{M}}=\Pi x \in \mathcal{M}$ and $x_{\mathcal{N}}=\left(\mathbf{I}_{\mathcal{L}}-\Pi\right) x \in \mathcal{N}$. If $x=x_{\mathcal{M}}^{\prime}+x_{\mathcal{N}}^{\prime}$ was any other such representation, then $x_{\mathcal{M}}=\Pi\left(x_{\mathcal{M}}^{\prime}+x_{\mathcal{N}}^{\prime}\right)=x_{\mathcal{M}}^{\prime}$. Hence, $x_{\mathcal{N}}=x_{\mathcal{N}}^{\prime}$ as well.

$(\Leftarrow)$. Since $\Pi_{\mathcal{M} \| \mathcal{N}}\left(x_{\mathcal{M}}+x_{\mathcal{N}}\right)=x_{\mathcal{M}}$ for any $x_{\mathcal{M}} \in \mathcal{M}$ and $x_{\mathcal{N}} \in \mathcal{N}, \Pi_{\mathcal{M} \| \mathcal{N}}$ is a parallel projection.

In the notation of the proposition given above, $\Pi_{\mathcal{M} \| \mathcal{N}}$ is called the parallel projection onto $\mathcal{M}$ along $\mathcal{N}$. Similarly for $\Pi_{\mathcal{N} \| \mathcal{M}}$ 
Before going on to the main result of the section we return to the feedback interconnection of Fig. 1. Let $\mathcal{G}_{\mathbf{P}}$ be the graph of the plant $\mathbf{P}$ and let

$$
\mathcal{G}_{\mathrm{C}}^{J}:=\left(\begin{array}{c}
\mathrm{C} \\
\mathrm{I}_{p}
\end{array}\right) \mathcal{D}_{\mathrm{C}}
$$

be the inverse graph of the controller. The following result relates stability with the idea of coordinatization. We remark that related results for nonlinear stability have been obtained in [20] (cf. [20, Proposition 4.2]).

Proposition 4. The feedback interconnection $[\mathbf{P}, \mathbf{C}]$ is stable if and only if $\mathcal{G}_{\mathbf{P}}, \mathcal{G}_{\mathbf{C}}^{I}$ induce a coordinatization of $\mathcal{L}=u \times \mathcal{Y}$.

Proof. See [1].

Propositions 3 and 4 together show that a stable fecdback configuration induces a pair of canonical parallel projections in the sense of Definition 2. These are precisely the operators $\Pi_{1}$ and $\Pi_{2}$ defined in the Introduction in (3). However it should be noted that the expressions given in (1) and (2) are not valid in the nonlinear case. An alternate expression for $\Pi_{1}$ which is valid in the nonlinear case is as follows (see [4]):

$$
\Pi_{1}:\left(\begin{array}{l}
d_{1} \\
d_{2}
\end{array}\right)-\left(\begin{array}{l}
u_{1} \\
y_{1}
\end{array}\right)
$$

where

$$
\left(\begin{array}{l}
u_{1} \\
y_{1}
\end{array}\right)=\left(\begin{array}{cc}
\mathbf{I}_{m} & \mathrm{O} \\
\mathrm{O} & -\mathrm{I}_{\gamma^{\prime}}
\end{array}\right)\left(\mathbf{F}_{\mathrm{P} \cdot \mathrm{C}}^{-1}-\left(\begin{array}{cc}
\mathrm{O} & \mathrm{O} \\
\mathrm{O} & \mathrm{I}_{\gamma^{\prime}}
\end{array}\right)\right)\left(\begin{array}{l}
d_{1} \\
d_{2}
\end{array}\right)
$$

A similar expression is available for $\Pi_{2}$. The relation (4) shows that properties of the feedback system (equivalently, of the input-to-error operator $\mathbf{F}_{\mathbf{P}, \mathrm{C}}^{-1}$ ) directly relate to analogous statements in terms of either of the two relevant parallel projections. We summarize below such a conclusion pertaining to the fg-stability of the feedback system.

Proposition 5. Let the feedback interconnection $[P, C]$ be stable, $\mathcal{M}:=\mathcal{G}_{\mathbf{P}}$, and $\mathcal{N}:=\mathcal{G}_{\mathrm{C}}^{I}$. Then, $[\mathbf{P}, \mathrm{C}]$ is fg-stable if and only if $\Pi_{\mathcal{M}|| \mathcal{N}}$ is fg-stable.

Proof. Follows from (4) and the definition of fg-stability. $\square$

In the context of linear systems the norm of the parallel projections has been related to the robustness of stability of the feedback system. In fact, in the linear case, the norm of the two parallel projections $\Pi_{\mathcal{M} \| \mathcal{N}}$ and $\Pi_{\mathcal{N} \| \mathcal{M}}$ are identical (see [9], [7]). The final result in this paper reports this fact for the case when at least one of the $\mathcal{M}$, $\mathcal{N}$ is a linear manifold (and the other possibly nonlinear). (The application to feedback systems takes $\mathbf{P} \in \mathcal{P}_{\mathcal{U}, \boldsymbol{y}}$, $\mathrm{C} \in \mathcal{P}_{\boldsymbol{y}, \mathcal{U}}$ with $[\mathbf{P}, \mathbf{C}]$ stable and $\mathcal{M}:=\mathcal{G}_{\mathbf{P}}, \mathcal{N}:=\mathcal{G}_{\mathbf{C}}^{I}$ as before, with either $\mathbf{P}$ or $\mathbf{C}$ a linear system.)

Proposition 6. Let $\mathcal{M}, \mathcal{N}$ be a coordinatization of $\mathcal{L}$ and suppose that $\Pi_{\mathcal{M} \| \mathcal{N}}$ is $\mathrm{fg}$-stable. If $\mathcal{M}$ is a linear manifold then

$$
\left\|\Pi_{\mathcal{M} \| \mathcal{N}}\right\|=\left\|\Pi_{\mathcal{N} \| \mathcal{M}}\right\| .
$$

Proof. See [4].

The proof given in [4] is reminiscent of a proof given in [14] to show that the norm of two complementary parallel projections are equal in the linear case. However, the precise argument in [14] translated to the case above fails in case one of the manifolds is nonlinear. At present we do not know whether for arbitrary manifolds $\mathcal{M}, \mathcal{N}$, which coordinatize $\mathcal{L}$, the two projections have equal norm, i.e., whether for nonzero $x_{\mathcal{M}}, x_{\mathcal{N}}$

$$
\sup _{x_{\mathcal{M}} \in \mathcal{M}, x_{\mathcal{N}} \in \mathcal{N}} \frac{\left\|x_{\mathcal{M}}\right\|}{\left\|x_{\mathcal{M}}+x_{\mathcal{N}}\right\|}=\sup _{x_{\mathcal{M}} \in \mathcal{M}, x_{\mathcal{N}} \in \mathcal{N}} \frac{\left\|x_{\mathcal{N}}\right\|}{\left\|x_{\mathcal{M}}+x_{\mathcal{N}}\right\|} .
$$

We conclude with an example discussed in [3].

Proposition 7. Let $\mathrm{P} \in \mathcal{P}_{u, y}$ a stable plant with $\langle\mathbf{P} u, u\rangle \geq 0$ for all $u \in \mathcal{U}$ (i.e., a passive system), $\mathbf{C}=-\mathbf{I}$, $\mathcal{M}=\mathcal{G}_{\mathbf{P}}$, and $\mathcal{N}=\mathcal{G}_{\mathbf{C}}^{l}$. Then,

(a) $[\mathbf{P}, \mathbf{C}]$ is fg-stable, and

(b) $\left\|\Pi_{\mathcal{M} \| \mathcal{N}}\right\|=\left\|\Pi_{\mathcal{N} \| \mathcal{M}}\right\| \leq \sqrt{2}$.

Proof. With $d_{1}, d_{2}, u_{1}, u_{2}, y_{1}, y_{2}$ as in Fig. 1, we calculate

$$
\begin{aligned}
2\left(\left\|d_{1}\right\|^{2}+\left\|d_{2}\right\|^{2}\right) & \geq\left\|d_{1}+d_{2}\right\|^{2} \\
& =\left\|u_{1}+y_{1}\right\|^{2} \\
& =\left\|u_{1}\right\|^{2}+\left\|y_{1}\right\|^{2}+2\left\langle u_{1}, y_{1}\right\rangle \\
& \geq\left\|u_{1}\right\|^{2}+\left\|y_{1}\right\|^{2} .
\end{aligned}
$$

Therefore,

$$
\frac{\left\|u_{1}\right\|^{2}+\left\|y_{1}\right\|^{2}}{\left\|d_{1}\right\|^{2}+\left\|d_{2}\right\|^{2}} \leq 2
$$

and the result follows from Proposition 6 since $\mathbf{C}$ is a linear system.

\section{References}

[1] S. Buddie, T.T. Georgiou, Ü. Özgüner and M.C. Smith, Flexible structure experiments at JPL and 
WPAFB: $H_{\infty}$ Controller Designs, Cambridge University Engineering Department Technical Report, CUED/F-INFENG/TF 99, 1992.

[2] W.N. Dale and M.C. Smith, Stabilizability and existence of system representations for discrete-time timevarying systems, Proceedings of the 1991 American Control Conference, Boston, June 1991, pp. 27372742.

[3] J. C. Doyle, Workshop presentation, Santa Barbara, CA, 1991

[4] J. C. Doyle, T. T. Georgiou, M. C. Smith, The parallel projection operators of a nonlinear feedback system, February 1992, preprint (submitted for publication).

[5] A. K. El-Sakkary, The gap metric: Robustness of stabilization of feedback systems, IEEE Trans. on Aur tomat. Contr., 30: 240-247, 1985.

[6] M.J. Englehart and M.C. Smith, A Four-block problem for $H_{\infty}$ design, Automatica, 1991, 27: 811-818.

[7] C. Foias, T.T. Georgiou, and M.C. Smith, Geometric techniques for robust stabilization of linear timevarying systems, Proceedings of the 1990 IEEE Conference on Decision and Control, pp. 2868-2873, December 1990, also in SIAM J. Control and Optimization, to appear.

[8] T.T. Georgiou, On the computation of the gap metric, Systems and Control Lclt., 11: 253-257, 1988.

[9] T.T. Georgiou and M.C. Smith, Optimal robustness in the gap metric, IEEE Trans. on Automat. Control, 35: $673-686,1990$.

[10] T.T. Georgiou and M.C. Smith, Robust control of feedhack systems with combined plant and controller uncertainty, Proceedings of the 1990 American Control Conference, pp. 2009-2013, May 1990.

[11] T.T. Georgiou and M.C. Smith, Linear systems and robustness: a graph point of view, Robust Control Workshop, Tokyo, June 1991, to be published by Birkhauser.

[12] K. Glover and D.C. McFarlane, Robust stabilization of normalized coprime factor plant descriptions with $H_{\infty}$-bounded uncertainty, IEEE Trans. on Automat. Ciontr., 34: 821-830, 1989.

[13] R.A. Hyde, The application of robust control to VSTOL aircraft, Ph.D. Thesis, University of Cambridge, August 1991.

[14] T. Kato, Estimation of iterated matrices, with application to the von Neumann condition, Numerische Mathematik, 2: 22-29, 1960.

[15] D.C. McFarlane and K. Glover, Robust controller design using normalized coprime factor plant descrip. tions, Lecture notes in Control and Information Sciences, Springer-Verlag, 1990.

[16] R.J. Ober and J.A. Sefton, Stability of control systems and graphs of linear systems, Systems and Control Lett., 17: 265-280, 1991.

[17] L. Qiu and E. J. Davison, Feedback stability under simultaneous gap metric uncertainties in plant and controller, Systems and Control Lcti., 18: 9-22, 1992.

[18] M. Safonov, Stability and Robustness of Multivariable Feedback System, MlT Press, 1980.
[19] J. M. Schumacher, A pointwise criterion for controller robustness, Systems and Contol Lett., 18: 1-8, 1992.

[20] M. S. Verma, Coprime fractional representations and stability of non-linear feedback systems, Int. J. Control, 48(3): 897-918, 1988.

[21] M. Vidyasagar, Input-output stability of a broad class of linear time-invariant multivariable feedback systems, SIAM J. Control, 10: 203-209, 1972.

[22] $M$. Vidyasagar, The graph metric for unstable plants and robustness estimates for feedback stability, IEEE Trans. on Automat. Contr., 29: 403-418, 1984.

[23] M. Vidyasagar and H. Kimura, Robust controllers for uncertain linear multivariable systems, Automatica, 22: 85-94, 1986.

[24] M. Vidyasagar, H. Schneider and B. Francis, Algebraic and topological aspects of feedback stabilization, IEEE Trans. on Automat. Contr., 27: 880-894, Aug. 1982.

[25] G. Vinnicombe, Structured uncertainty and graph topology, Cambridge University Engineering Department Techuical Report, CUED/F-INFENG/TF 76, 1991.

[26] G. Zames, On the input-output stability of timevarying nonlinear feedback systems. Part I: Conditions using concepts of loop gain, conicity, and positivity, IEEE Trans. on Autom. Control, 11(2): 228$238,1966$.

[27] G. Zames and A. K. El-Sakkary, Unstable systems and feedback: The gap metric, Proc. of the Allerton Conference, pp. 380-385, 1980.

[28] S.Q. Zhu, M.L.J. Hautus and C. Praagman, Sufficient conditions for robust. BIBO stabilization: Given by the gap metric, Systems and Control Letters, 11: 5359,1988 . 